\title{
A Chaotic Salp Swarm Feature Selection Algorithm for Apple and Tomato Plant Leaf Disease Detection
}

\author{
S.Venkatasubramanian ${ }^{1}$ \\ ${ }^{1}$ Associate Professor, Saranathan college of Engineering, Trichy, India,620012, veeyes@saranathan.ac.in \\ Received Date : August 06, 2021 Accepted Date : September 15, $2021 \quad$ Published Date : October 06, 2021
}

\begin{abstract}
Plants are seen as vital because they provide mankind with energy. Plant diseases can harm the leaf at any time between planting and harvesting, resulting in enormous losses in crop output and market value. A leaf disease detection system acts asignificant role in agricultural production. A large amount of labour is required for this process as well as an in-depth understanding of plant diseases. Determining the presence of illnesses in plant leaves requires the use of deep learning and machine learning methods, which classify the data based on a specified set. In this paper, apple and tomato leaves disease detection process is carried out by Chaotic Salp Swarm algorithm (CSSA) followed by Bi-directional Long Short Term Memory (Bi-LSTM) technique for classification. We've used the Bi-LSTM architecture to sense disease in tomato and apple leaves in studies. In order to determine the type of leaves, we trained a deep learning network using the PlantVillage dataset of damaged and healthy plant leaves. It is estimated that the trained model achieves a test accuracy of $96 \%$.
\end{abstract}

Key words: Apple Leaves; Chaotic Salp Swarm algorithm; Deep Learning Technique; PlantVillage Dataset; Tomato Leaves.

\section{INTRODUCTION}

If left ignored, disease in agricultural crops can have a significant influence on the economy, yield, and output. Diseases that are accurately detected and discovered at an early stage can be controlled with pesticides or their equivalents. Plant symptoms can be determined by a variety of on-the-ground and laboratory investigations, including soil analysis and pH monitoring. This is a time-consuming, expensive, and tiresome task. Diverse ICT and AI-based decision support systems are being created to assist existing farmers and the next generation of farmers ([1-2]).

Increased agriculture production in India is vital for the country's economic progress. Plant growth is a key factor in agricultural production. Among the factors that influence plant growth are light, water, nutrients, minerals, and hotness. It is the leaves of plants that carry out the photosynthesis course and exchange of water with the outside world. Scientists rely on leaf measurements (leaf area, leaf circumference, leaf maximum length, and leaf maximum width) to analyze plant biological features. Thus measuring these values quickly and properly is crucial. Leaf region is an irregularly shaped portion on one side of the leaf. It symbolizes a plant's ability to produce oxygen through photosynthesis. The camera's image analysis relies heavily on the image processing algorithm. For leaf extentcapacities, traditional methods include graph paper grid counting, and regression equation method. Despite their simplicity, these approaches have the disadvantages of limited precision and high costs [4]. Despite the excellent accuracy of the various Leaf area meter, the large leaf must be broken into minor segments for measurement, which can lead to mistakes.

As the name suggests, plant infection is an aberrant state of a plant that interferes with its growth [4]. Different forms of illnesses can cause damage to plant leaves. Disease identification and classification are crucial. Leaf diseases have a negative impact on crop productivity. Prevention and control can be achieved through early detection and proper action to limit losses. Experienced farmers can spot these diseases with their naked eyes, however there are restrictions according to a person's physical state such as weariness, eyesight and working conditions. If any of these errors occur, the result could be incorrect. Foliar illnesses can be identified by a machine, which can help the farmer monitor plant growth and boost productivity [6-8]. As it shows on plant leaves, it also aids in early disease detection [8].

It is possible to detect and categorize different leaf diseases by using image processing stages such as acquisition, filtering or segmentation as well as image feature extraction. [9-11] Viruses, fungi, and bacteria are the principal causes of leaf diseases. Sustainable development is primarily concerned with reducing pesticide use, protecting the 
environment, and improving produce quality. An early, accurate, and precise diagnosis decreases pesticide use. Today, LSTMs are commonly utilized for image classification. In this paper, segmentation, feature selection and classification are the major steps for apple and tomato leaves disease detection. A modified Fuzzy C-Means (FCM) is used for segmentation, CSSA for feature selection and BiLSTM for classification are used in this research study. The remaining paper is consists of related works are presented in Section 2. The explanation of the proposed work is given in Section 3, where the validation results of proposed method with existing techniques are delivered in Section 4. Finally, the conclusion of the research work along with future work is obtainable in Section 5 .

\section{LITERATURE REVIEW}

Using CNN, Lucas G. Nachtigall et al. [12] classified and detected illnesses, nutritional deficits, and apple tree herbicide damage from leaf pictures. If the quality of yields can be improved by involving more experts in the field, it could reduce scale and increase costs. In a study using a real dataset of 2539 photos from six different illnesses, the researchers found that the trained CNN outperformed the untrained one, with a 97.3 percent accuracy rate.

An apple leaf disease identification method was developed by Zhang Chuanlei et al. [13]. Using SVM, these disorders can be identified. The selected subset of features is universally optimal in the suggested technique. On the basis of the simulation results, 90 percent of the apple disease leaf illustrations can be correctly identified, indicating that the proposed work model is both effectual and feasible.

With the use of detection and classification techniques, Barbedo [14] has evaluated the image of the sick leaf. In this study, a method for automatically segmenting disease signs from plant leaves is presented. To alter the sick leaf's color, Boolean operations have been used. On the basis of a leaf disease database, the proposed approach was evaluated.

Researchers Kalaivani et al. [15] have focused on the segmentation strategies to progress the accuracy rate and protect the leaf from a variety of ailments. Region of interest (ROI) of input image segmented, and histogram created. As a result of the segmentation method, the diseased area of the plant can be identified. A variety of validation techniques and similarity measurements were also employed in order to improve the results even further The created approach has a 98 percent accuracy rate, while the approximate similarity measure is only 1 percent.

Using hyperspectral data, Golhani et al. [16] propose an article based on advanced NN. As a result, researchers offered an overview of hyperspectral data processing using neural networks $(\mathrm{NN})$. Analyzing the data revealed the plant's early sickness. The hyperspectral data and the NN mechanism have also been linked through hybridization. The Spectral Disease Index appears to be a useful tool for detecting the disease using the suggested approach (SDI).

Agricultural researchers Salazar-Reque et al. [17] have presented a revolutionary crop protection strategy. Though time-consuming and extremely subjective, automation is eagerly expected in this area. Simple Linear Iterative Clustering (SLIC) is used to group superpixels together. In order to classify superpixel clusters as healthy or unhealthy, artificial neural networks (ANNs) were trained using the color attributes of the superpixel clusters. These parameters of the network are heuristic in nature. Using visual groups and plants, the classifier's performance was assessed. The average value of F-score is 0.67 , which was higher than earlier methods.

Hybrid clustering [18] involves breaking the image into many compacts and using those as clustering signals for the super-pixel clustering. Following the picture segmentation, expectation maximization (EM) method is used to quickly and correctly separate lesion pixels from super-pixels. As a consequence of the experimental and comparative results, the proposed method is highly effective.

Dr. Paul P. Mathai, et.al. [24], used support vector machine to detect the diseased leaves in plants. SVM classifier is used to classify the image as healthy or unhealthy.

\section{PROPOSED METHODOLOGY}

In this section, the description of proposed approach in each step is presented. Figure 1 shows the workflow of proposed methodology. Two leaves datasets are taken as input, where raw data is modified using pre-processing. CSSA is used for selecting the relevant features and Bi-LSTM classifier is used for final prediction.

\subsection{Dataset Description}

The proposed system has been analysed with the help of two different types of data sets such as Apple plant village dataset and Tomato plant village Datasets. Both dataset are detail described in the below section

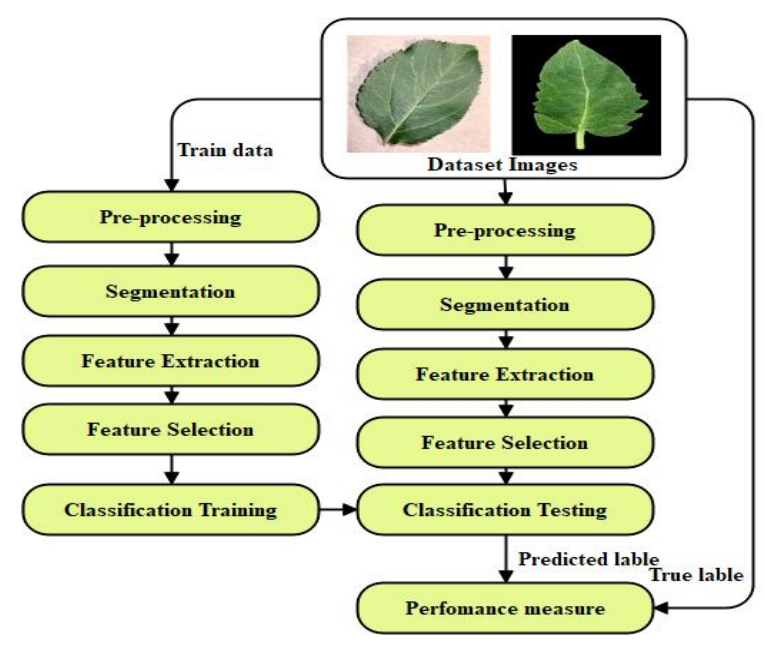

Figure 1: Working Flow of proposed methodology

\subsubsection{Apple plant village Dataset}

The dataset used in this project consists of four prominent classes, where three are diseases and one is the class of 
healthy leaves. We have 1000 samples of healthy leaf images and 1526 samples of diseased leaf images. This dataset is a subset of the popular Plant Village dataset. For a specific image of apple leaf, classification technique is required, which can classify input images into one of the classes as shown below in Figure 2.
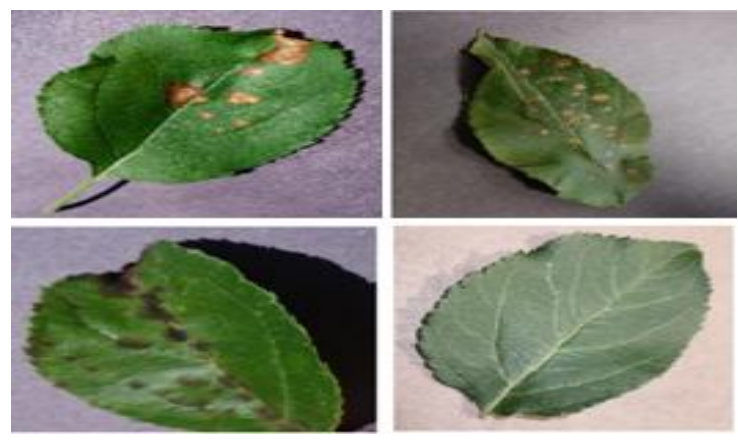

Figure 2: Sample Images of Apple leaf with its disease.

\subsubsection{Tomato plant village Datasets}

The Plant Village library was used to find photographs of tomato leaf disease. Using a python script, images of the diseases were downloaded [19]. It is estimated that the gathered dataset contains roughly 18160 photos, which are divided into ten different categories. All of the primary leaf diseases that potentially harm tomato crops are represented in this collection of photos. Each of the downloaded photos was stored in the uncompressed JPG format and defaulted to the RGB color space. The sample image of tomato leaves in Figure 3 is shown below.
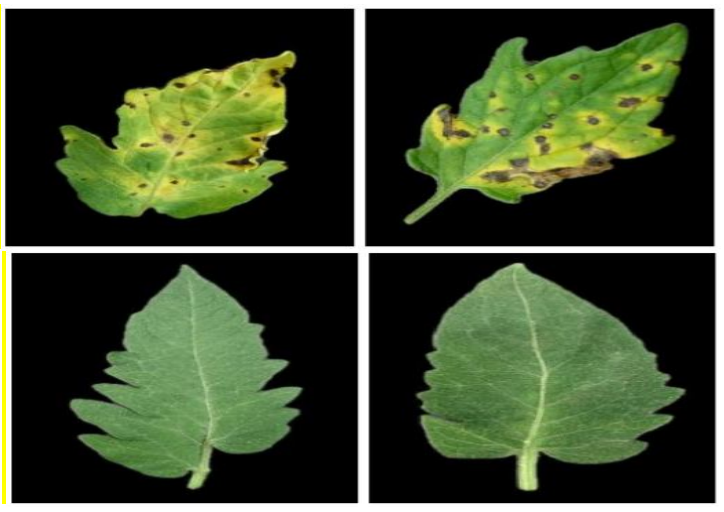

Figure. 3: Sample Images of Tomato Leaves with its diseases

\subsection{Image Pre-processing}

Images of apple and tomato leaves will be commonly acquired in RGB color space model. Color is an important feature as color value shows variations based on disease infection in the plant. Other color space models like RGB, HSI, CMYK, L*a*b and HSV can also be used for analysis. Acquired images are manipulated directly in RGB color space model and color transformation is performed over the image to standardize the color model. HSI and HSV color space models are commonly used in leaf disease identification as they are similar to human perception. In HSI color model Hue, and Intensity of a color is observed and in HSV color model Hue, and Value of a color is considered for manipulation. Hue is an important color component taken into consideration as it shows the perceived color of an object.

During image pre-processing, an image is altered to provide more information about the affected area of a leaf. With good visual interpretation, it presentsleaf image information effectively. This procedure does not modify the leaf image's inherent information, but it does change the dynamic range of specific aspects for localization, allowing for a more accurate localization. Image resizing and filtering are the common pre-processing techniques used in apple and tomato leaves disease diagnosis. Captured leaf image in different resolution sizes are standardized to a fixed resolution size using image resizing. Image filtering is used to remove unwanted region in leaf images as there are more possibilities to have dust particles due drops in leaf area. Filtering process is performed either as low pass or high pass filter. Low pass filter lessens the amplitude of high frequencies and has low frequencies unchanged. High pass filter retains high frequencies and soothes the amplitude of low frequencies. Median filter and Average filter are commonly used filtering techniques to reduce noise in an image. The pre-processed images are given to the segmentation which is described in the below section.

\subsection{Segmentation}

As a result, digital noise and high-frequency components can be removed from the input images via filtering. For particular purposes, digital radiographs are enhanced and restored to produce a more acceptable output. Images are smoothed using two-dimensional filters, sharpened using edges and contrast stretched. To recognize metal objects, edge segmentation is critical since edges of metal objects photographs store a lot of information about the input leaf images. Adaptively regularized kernel-based FCM framework (AR-KFCM) is used for segmentation in this method. For each pixel in this system, we first calculate the Flexible parameter $\varphi_{a}$ connected with it in order to manipulate the contextual information by employing (1). When defining the objective function, we say that

$$
\begin{aligned}
& J_{\mathrm{ARKFCM}}=2\left[\sum_{a=1}^{i} \sum_{b=1}^{c} u_{a b}^{m}\left(1-K\left(m_{a}, v_{b}\right)\right)+\right. \\
& \left.\sum_{a=1}^{i} \sum_{b=1}^{c} \varphi_{a} u_{a b}^{m}\left(1-K\left(\bar{m}_{a}, v_{b}\right)\right)\right]
\end{aligned}
$$

The minimization of $J_{\mathrm{KFCM}}(m, v)$ can be calculated through an substitute optimization process using

$u_{i j}=\frac{\left(\left(1-K\left(m_{a}, v_{b}\right)\right)+\varphi_{a}\left(1-K\left(\bar{m}_{a}, v_{j}\right)\right)^{-1 /(m-1)}\right.}{\left.\sum_{a=1}^{N} u_{a, b}^{m}\left(K\left(m_{a}, v_{j}\right)+\varphi_{a}\left(1-K\left(\bar{m}_{a}, v_{j}\right)\right)\right)^{-1 /(m-1}\right)}$ 
$v_{a}=\frac{\sum_{a=1}^{N} u_{a, b}^{m}\left(K\left(m_{a}, v_{b}\right) m_{a}+\varphi_{a} K\left(\bar{m}_{a}, v_{j}\right) \bar{m}_{a}\right)}{\sum_{a=1}^{N} u_{a, b}^{m}\left(K\left(m_{a}, v_{b}\right) m_{a}+\varphi_{a} K\left(\bar{m}_{a}, v_{j}\right)\right)}$

When $\bar{x}$ is replaced with average filter of the input leaves image into grayscale, the algorithm is denoted as ARKFCM. When $\bar{x}_{i}$ is replaced with the weight image $\bar{\varepsilon}_{i}$ defined. The algorithm is denoted as $\operatorname{ARKFCM}_{\omega}$. The chief step for the ARKFCM is stated below:

\section{ARKFCM algorithm}

(1) Initialized threshold $\varepsilon=0.001, m=2$, loop counter $t=0, v$ and $u^{(0)}$

(2) Compute the regularization parameter $\varphi_{i}$.

(3) Compute $\bar{x}_{i}$ for ARKFCM

(4) Compute cluster centers $v_{j}^{(t)}$ using $u^{(t)}$ as in (3).

(5) Compute the membership function $u^{(t+1)}$ within (2)

(6) If $\max || u^{(t+1)}-u^{t} \mid<\varepsilon$ or $t>100$ then stop; otherwise, update $t=t+1$ and go to step (2).

\subsection{Feature extraction}

A square-shaped function is utilized to compute DWT's wavelet coefficients for the input images. Short support filters, low processing, and no redundancy make the DWT approach more efficient in terms of compression energy, as well as reconstruction. When using the fuzzy de-noising technique, the DWT produces sub-bands that are capable of being shifted as well as greater directional selectivity with less redundancy Zooming in and out is used to calculate the true texture of the image in the multi-resolution process. To preserve the low and high frequency information, the images are usually fragmented into aamount of sub-images at varying resolution levels. In order to extract the texture information from photos, you can use the DWT property. In the inner product of the wavelet transform $\mathrm{w}$ and the square integral function $f(u)$, the inner product $\mathrm{f}$ represents the original valued function $\psi(u)$ In the equation, the wavelet function is given (5).

$$
w[f(s, \tau)]=\left(f, \psi_{s, t}^{k}\right)=\int_{\infty}^{\infty} f(u) \psi_{s, t}^{k}(u) d u
$$

Where, $\psi_{s, t}^{k}(u)=\left(\frac{1}{\sqrt{s \psi_{s, t}^{k}}} / s\right)$ denotes

wave family, $s \in Z$ is scale, $\tau$ is translation and $k \in\{h, v, d\}$ is orientation factors. The orientation parameters $h, v$ and $d$ denote vertical, horizontal and diagonal direction respectively. There are two ways to decompose a dyadic wavelet. Dwt dyadic wavelet decomposition is a scalable sample of DWT. Using perfect reconstruction filter banks, the uses dyadic wavelets. Eq. (6) shows that the wavelet function $\psi(u)$ and the scaling function $\varphi(u)$ can be used (7). The wavelet atoms are described by three mother atoms and scaling $\psi^{h}, \psi^{v}$ and $\psi^{d}$. This mother atoms computed as the tensor products of 1-dimensional $\psi(u) \operatorname{and} \varphi(u)$, that is signified in Eq. (8) and (9)

$$
\begin{aligned}
& \psi_{j, n}^{k}(u)=\frac{1}{\sqrt{2^{j}}} \psi^{k}\left(\frac{u-2^{j} \cdot n}{2^{j}}\right) \\
& \varphi_{j, n}^{k}(u)=\frac{1}{\sqrt{2^{j}}} \psi^{k}\left(\frac{u-2^{j} \cdot n}{2^{j}}\right) \\
& \varphi(u)=\varphi\left(u_{1}\right) \varphi\left(u_{2}\right), \psi^{k}(u) \\
& \varphi^{v}(u)=\varphi\left(u_{1}\right) \psi\left(u_{2}\right), \psi^{d}(u)
\end{aligned}
$$

Down samplers and digital filter banks are used to implement the two-dimensional DWT. Low-pass and highpass filters comprise the digital filter bank. In the wavelet configuration structure, the bank numbers are arranged according to the intended functions. In order to construct a 2dimensional wavelet coefficient, the rows and columns of the underwater image are independently transformed using the 1-dimensional wavelet transform. The original images $A_{2^{j+1}} f$ at resolution $2^{j+1}$ decompose into 4 -subband images in the frequency domain. Three sub-band images such as $D_{2}^{h} f, D_{2^{i}}^{h} f$ and $D_{2^{i}}^{h} f$ are the original images in resolution in vertical, horizontal and diagonal, $A_{2} i f$ found at coarse resolution, so the entire under water image $A_{2}{ }^{j+1} f$ is denoted in the Eq. (10).

$A_{2^{j+1}} f=D_{2^{i}}^{h} f,+D_{2^{i}}^{v} f+D_{2^{i}}^{d} f+A_{2^{i}} f$

The decomposed sub-images are the 2-dimensional orthogonal wavelet. The 4-orthogonal sub-bands such as Low-Low (LL) band, Low-High (LH) band, High-Low (HL) band and High-High $(\mathrm{HH})$ band, which is represented as $D_{2^{i}}^{h} f,+D_{2^{i}}^{v} f+D_{2^{i}}^{d}$ fand $_{2^{i}} f \quad$ respectively. Wavelet highlights are extracted from each sectioned picture based on the LL phase of component extraction. This can be done by utilizing wavelet highlights and GLCM (Gray Level CoEvent Matrix). As an example, highlights register's us cluster prominence, cluster shade and dissimilarity are all examples of autocorrelation; contrast is an example of contrast; homogeneity, homogeneity is an example of homogeneity; Probability at its highest level The number of cubes, Variance, entropy, difference fluctuation, transformation entropy and sum normal. Correlation proportion of evidence1, correlation proportion of information2, A standardized Inverse distinction, Inverse contrast, and Converse alteration instant

\subsection{The novel chaotic salp swarm algorithm}

Here in, a CSSA is proposed, whereby chaos-mapped random variables are substituted for random ones in the CSSA. When it comes to the original, its performance is influenced by three factors. This is a list of parameters $\mathrm{c} 1$ through c3. C1 decreases linearly during the iterations, while c3 determines whether the next position should be toward 
negative or positive infinity, as illustrated by [20]. C2 and $\mathrm{c} 1$ are the two key characteristics that influence a salp's updating position, as shown in [20]. Consequently, they have a considerable impact on the balancing act between exploration and extraction whereas exploration focuses on finding new, better solutions by examining the search space on a global scale, the focus of exploitation is on using the data in a narrow area, It is important that an algorithm balances these two factors in order to approximate the global optimum. SSA c2 parameter is adjusted using chaotic maps in this study. According to the chaotic map, equation (11) updates the c2 parameter. On the other hand, equation (12) displays the salp's updated position in relation to the chaotic map.

$$
\begin{gathered}
c 2=o_{s} \\
y_{i}^{t}=\left\{\begin{array}{c}
\left.F_{i}+c_{1}\left(\left(u b_{i}-l b_{i}\right)\right) o^{t}+l b_{i}\right), c_{3} \geq 0 \\
\left.F_{i}-c_{1}\left(\left(u b_{i}-l b_{i}\right)\right) o^{t}+l b_{i}\right) \quad c_{3}<0
\end{array}\right.
\end{gathered}
$$

The performance and convergence rate of SSA can be improved by incorporating chaotic maps within the salp updating position. A discrete binary solution pool is used in CSSA feature selection method, where salp positions are limited to $0,1^{\prime}$. Assume an Ith-dimensional variable $\mathrm{y}$ represents a salp position. $y=\left[x_{1}, x_{2}, \ldots, x_{\mathrm{dim}}\right]$, what's a $\mathrm{dim}$ the maximum number of dimensions is dim This signifies that a feature has been selected when the variable value is 1 , and that it has not been selected if the variable value is 0 . Data with 5 features can be represented as [1,0,0,1] or $[1,0,1,1,1]$ and so on. There are a variety of characteristics and lengths in the two previous samples. A random numeral in the range $[0,1]$ is used in equation (13) to explain how each agent moves from continuous to discrete binary space.

$$
y_{i}^{t}\left\{\begin{array}{c}
1 \quad \text { if }\left(s\left(y_{i}^{t}\right)\right) \geq B \\
0 \text { otherwise }
\end{array}\right.
$$

Where

$$
s\left(y_{i}^{t}\right)=\frac{1}{1+e^{10\left(y_{i}^{t}-0.5\right)}}
$$

The following is a description of the suggested chaotic variant of the SSA algorithm:-

\subsubsection{Parameters initialization}

CSSA starts with salps positions that are randomly generated. This is followed by a collection of initial parameters. The lower and higher boundaries are originally defined based on the benchmark function, while the lower and upper boundaries are initially established for the benchmark dataset. There is a limit of 500 iterations for global optimization and 30 for feature selection. Finally, for the global optimization problem, the population size is set to 50 , and for the feature selection problem, it is 20 . This is because the search space is so complex that the iterations is set low.

\subsubsection{Fitness function}

Each solution is evaluated based on the fitness function (salp position). All of the global benchmark issues that are employed involve minimization. Since the fitness value $\min (f(X))$ is the lowest, it's selected as the best option thus far. For example, when it comes to feature selection, the ideal approach is the one that increases classification accuracy while reducing the number of chosen characteristics. As shown in Equation (15), the fitness function employed for the feature selection issue combines these two objectives using a weight factor. KNN classifier's classification accuracy is Acc in this equation, where $\mathrm{k}$ is equal to 3 with mean absolute distance. It is a supervised machine learning approach that uses a KNN network. As a result of this, it classifies the new instance according to its distance from the training instances. This work uses a KNN classifier in order to determine the quality of the specified feature subsets that have been selected. When it comes to $\mathrm{K}$ and the distance method, it's a case of trial and error. When weight factor $w_{f}$ is utilized. Firstly, we want to exploit classification accuracy, then reduce the number of features used. As a result, $\mathrm{w} f$ is 0.8 . In the case of a given dataset, $\mathrm{Lt}$ represents the total number of features.

$F_{n_{t}}=\operatorname{maximize}\left(w f \times A c c+\left(1-w_{f}\right) \times\left(1-\frac{L_{f}}{L_{t}}\right)\right)$

\subsubsection{Positions updating}

Following the evaluation of each salp's fitness function, and selection of the optimal salp position According to the optimal salp position (12), (13), (3.3) and (3.4) from [20].

\subsubsection{Termination criteria}

There will be an infinite number of iterations of assessing and updating each salp's location until it extents the maximum total of iterations, or until the optimal solution is initiate. There's a limit number of iterations for global optimization and 30 for feature selection in our situation.

The proposed CSSA pseudo code is defined at Algorithm 2.

1. Set the initial value of maximum number of iterations $t$ Max, upper boundary $u b$, and lower boundary $l b$ population size $n$, number of dimensions dim and define the fitness function $F n$.

2. Randomly initialize the positions of salps $y_{i}^{t}$ corresponding to $u b$ and $l b$ where $i=1,2, \ldots$ nand $t=1,2, \ldots t$ Max.

3. Set $t:=1$ \{Counter Initialization $\}$.

4. Repeat.

5. Evaluate the fitness function of each salpposition $F n\left(y_{i}^{r}\right)$. 
6. Set the best salp position to ybest.

7. Update $c 1$ according to (3.2 from [20])

8. Get the value of chaotic map $o_{t}$

9. Update $c_{2}$ according to (11)

10. For $(i=1: i \leq n)$ do

11. If $i==1$ then

12. Update the position of the leading salp according to $(12)$

13. Else

14. Update the position of the follower salp according to (3.4 from [20])

15. End if

16. Update the position of the supporter salp according to (13)

17. End for

18. Check the feasibility of $y_{i}^{t}$

19. Evaluate the new position of crow $y_{i}^{t}$

20. If $F n\left(y_{i}^{t}\right)$ is better that $F n$ (ybest) then

21. Update the position of the best salp.

22. End if

23. Set $t=1+1$ \{iteration counter increasing $\}$.

24. Until $(t<t \max )$. \{Termination criteria satisfied .

Produce the best salp position tbest

\subsection{Classification}

Here, two classifiers such as LSTM and Bi-LSTM are used for final prediction. Each classifier is described as below:

\subsubsection{Long Short-term Memory (LSTM)}

RNN is the neural feedback network's extension. The gradient disappears or explodes, however, in the ordinary RNN. Long Shortened Memory Network (LSTM) has been designed to resolve the problems and has been performed superiorly. Three gates and one cell memory state exist in the LSTM architecture. Figure 4 appearances the LSTM standard architecture.

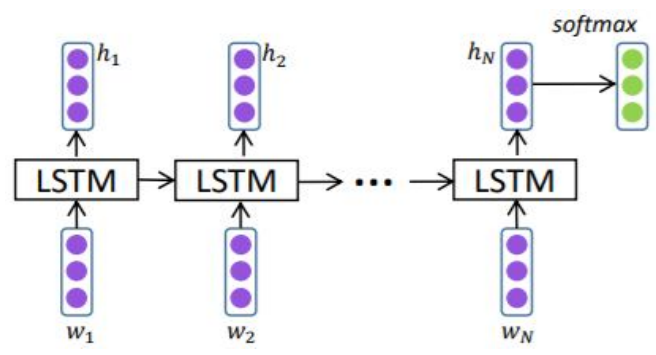

Figure. 4. The architecture of a standard LSTM.

$$
\begin{gathered}
X=\left[\begin{array}{c}
h_{t-1} \\
x_{t}
\end{array}\right] \\
f_{t}=\sigma\left(W_{f} \cdot X\right)+b_{f} \\
i_{t}=\sigma\left(W_{i} \cdot X\right)+b_{i}
\end{gathered}
$$

$$
\begin{gathered}
o_{t}=\left(W_{o} \cdot X\right)+b_{o} \\
c_{t}=f_{t} \odot c_{t-1}+i_{t} \odot \tanh \left(W_{c} \cdot X+b_{c}\right)(20) \\
h_{t}=o_{t} \odot \tanh \left(c_{t}\right)(21)
\end{gathered}
$$

where $W_{i}, W_{f}, W_{o} \in \mathbb{R}^{\times 2 \mathrm{~d}}$ dare the weighted matrices and $b_{i}, b_{f}, b_{o} \in \mathbb{R}^{\mathrm{d}}$ are biases of LSTM, which is the short-term memory solution. They have inbuilt systems, which can control the flow of information, called gates. These gates can find out which data to keep or throw away in one sequence. This enables it over the extended chain of sequences to transmit relevant information to make forecasts of tasks. The main notion of LSTM is the cell condition and its different gates. The cell state serves as a transportation route throughout the flow of information. During processing of the sequence, the cell state can contain useful data. Even early knowledge can thus lead to later temporal stages, which reduce the impact of short-term memory. The information will be added to or withdrawn by gates as the cell state moves on its trip. The gates can find out which knowledge is important in training to remember or forget.

\subsubsection{Bidirectional LSTM}

Because of the Bi-LSTM, the input sequence is sent to the LSTM in two different ways, resulting in two concurrent blocks of Bidirectional Long Short Term Memory. There are four gates: an input gate, an output gate, a forget gate, and a cell that make up an LSTM. Each of the two parallel bidirectional LSTM blocks we used has 112 units. When it came to hidden units, we employed sigmoid and Tanh, respectively.

\section{RESULTS AND DISCUSSION}

An Intel i3 3.0GHz processor, 1TB hard drive, and 8GB of RAM were used to test the proposed system. It is compared to existing schemes using a publicly accessible dataset in order to determine its effectiveness.

\subsection{Evaluation Metrics}

The challenge assessment metrics are used to assess our method's performance in segmentation and classification. For the evaluation criteria comprise sensitivity (SE), specificity (SP), Recall (R) accuracy (AC), and Precision (P). The performance criteria are distinct is as:

$$
\begin{gathered}
\text { Accuracy }=\frac{T P+T N}{T P+T N+F P+F N} \\
\text { Recall }=\frac{T P}{T P+F N} \\
\text { Precision }=\frac{T P}{T P+F N} \\
F-\text { Score }=\frac{2 * \text { Precision } * \text { Recall }}{\text { Precision }+ \text { Recall }}
\end{gathered}
$$


Where $t p, t n, f p$ and $f n$ denote the number of a true positive, true negative, false positive and false negative.

\subsection{Performance Analysis of Proposed CSSA with Deep Learning Technique}

Here, two types of analysis are carried out using proposed technique with existing techniques. The first analysis is presented using apple leave images and the validated results are given in Table 1 and graphical representation is described in Figure 5.

Table 1: Comparative Analysis of Proposed Feature Selection \& Classifier Technique

\begin{tabular}{|l|c|c|c|c|}
\hline \multirow{1}{*}{$\begin{array}{c}\text { Feature } \\
\text { Selection } \\
\text { Methodology }\end{array}$} & $\begin{array}{c}\text { Accuracy } \\
(\boldsymbol{\%})\end{array}$ & $\begin{array}{c}\text { Precision } \\
(\boldsymbol{\%})\end{array}$ & $\begin{array}{c}\text { Recall } \\
(\boldsymbol{\%})\end{array}$ & $\begin{array}{c}\text { F- } \\
\text { measure } \\
(\boldsymbol{\%})\end{array}$ \\
\hline PSO-LSTM & 88.89 & 79.12 & 80.92 & 85.27 \\
\hline PSO-Bi-LSTM & 72.32 & 80.53 & 83.69 & 86.07 \\
\hline $\begin{array}{l}\text { Salp Swarm- } \\
\text { LSTM }\end{array}$ & 81.43 & 82.07 & 90.06 & 89.28 \\
\hline $\begin{array}{l}\text { Salp Swarm-Bi } \\
\text { LSTM }\end{array}$ & 87.16 & 81.04 & 84.17 & 83.08 \\
\hline CSSA-LSTM & 94.38 & 95.43 & 96.46 & 96.34 \\
\hline $\begin{array}{l}\text { CSSA-Bi } \\
\text { LSTM }\end{array}$ & 96.90 & 97.84 & 98.20 & 98.67 \\
\hline
\end{tabular}

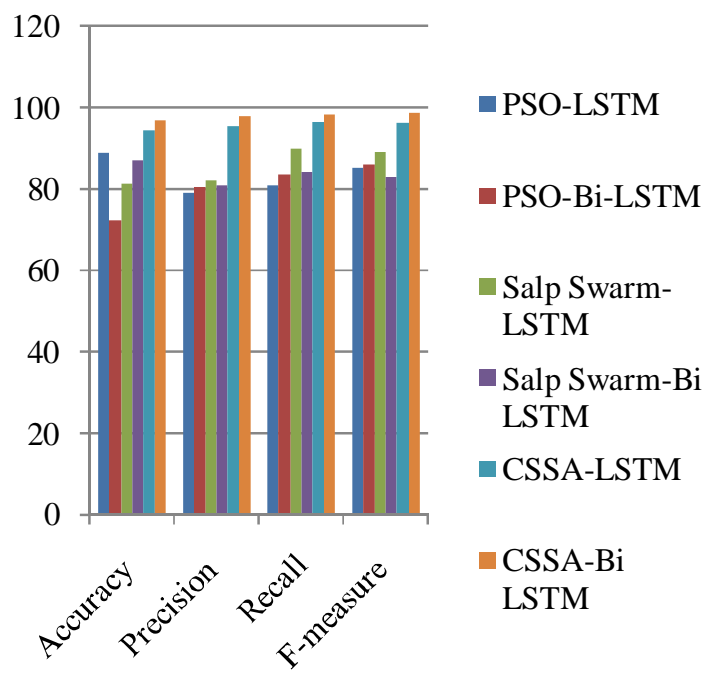

Figure 5: Graphical Representation of Proposed Technique for Apple Leaves Disease Detection

The existing technique called PSO is compared with our proposed classifiers and tested on the input images. The PSO with LSTM and Bi-LSTM achieved nearly $85 \%$ of accuracy, precision, recall and F-measure. The SSA with LSTM and Bi-LSTM achieved nearly $85 \%$ to $90 \%$ of accuracy, precision, recall and F-measure. But, our proposed CSSA with LSTM and Bi-LSTM achieved nearly $95 \%$ to $98.50 \%$ of accuracy, precision, recall and F-measure. This shows that
CSSA-Bi-LSTM achieved better performance than other existing techniques. The next Table 2 and Figure 6 displays the comparative examination of proposed technique for tomato leaves.

Table 2: Performance Analysis of Proposed Technique for Tomato Leave Disease Detection

\begin{tabular}{|l|c|c|c|c|}
\hline \multicolumn{1}{|c|}{$\begin{array}{c}\text { Feature } \\
\text { Selection } \\
\text { Methodology }\end{array}$} & $\begin{array}{c}\text { Accuracy } \\
(\boldsymbol{\%})\end{array}$ & $\begin{array}{c}\text { Precision } \\
(\mathbf{\%})\end{array}$ & $\begin{array}{c}\text { Recall } \\
\mathbf{( \% )}\end{array}$ & $\begin{array}{c}\text { F- } \\
\text { measure } \\
(\boldsymbol{\%})\end{array}$ \\
\hline PSO-LSTM & 85.22 & 78.48 & 84.78 & 89.73 \\
\hline $\begin{array}{l}\text { PSO-Bi- } \\
\text { LSTM }\end{array}$ & 87.45 & 82.73 & 88.36 & 88.46 \\
\hline $\begin{array}{l}\text { Salp Swarm- } \\
\text { LSTM }\end{array}$ & 79.86 & 82.07 & 90.06 & 91.45 \\
\hline $\begin{array}{l}\text { Salp Swarm- } \\
\text { Bi LSTM }\end{array}$ & 84.64 & 83.63 & 91.67 & 85.33 \\
\hline CSSA-LSTM & 92.09 & 93.43 & 94.98 & 95.63 \\
\hline $\begin{array}{l}\text { CSSA-Bi } \\
\text { LSTM }\end{array}$ & 96.59 & 96.45 & 97.44 & 98.34 \\
\hline
\end{tabular}

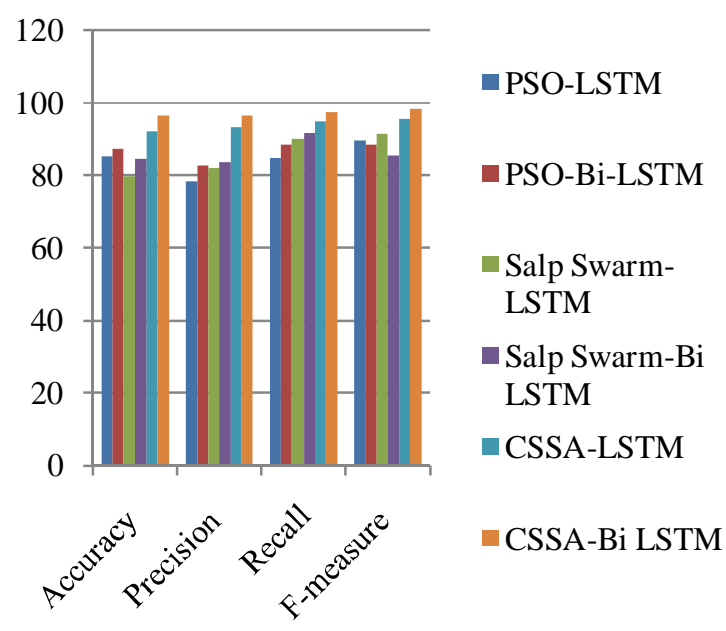

Figure 6: Graphical Representation of Proposed Technique for Tomato Leaves Disease Detection

When implementing with LSTM, PSO achieved nearly 79\% to $85 \%$ of all parameters, Salp Swarm achieved nearly $81 \%$ of accuracy and precision and CSSA achieved nearly $94.50 \%$ of all parameters. However, these techniques achieved better performance, while implementing with BiLSTM. For instance, PSO achieved $87 \%$ of accuracy, Salp Swarm achieved $84 \%$ of accuracy and proposed CSSA achieved $96.59 \%$ of accuracy. Therefore, CSSA with BiLSTM only achieved better performance than other techniques with LSTM.

\subsection{Comparative Analysis of Proposed Technique with overall disease detection}

In this section, the proposed technique (CSSA-Bi-LSTM) is compared with existing techniques in terms of accuracy. 
Table 3 and Figure 7 shows the comparative analysis of proposed technique with existing systems.

Table 3: Overall Comparative analysis of CSSA-Bi-LSTM with existing techniques

\begin{tabular}{|c|c|c|}
\hline Technique & Year & Accuracy (\%) \\
\hline LucasG. Nachtigallet al. [12] & 2016 & 96.16 \\
\hline Zhang Chuanleiet al. [13] & 2017 & 94.22 \\
\hline Tm, Prajwala et.al [21] & 2018 & 95.32 \\
\hline Bi, C et.al [22] & 2020 & 75.59 \\
\hline ChutinanTrongtorkid [23] & 2018 & 89.92 \\
\hline Pooja V et. Al [10] & 2017 & 92.4 \\
\hline Proposed_Apple leaf & 2021 & 96.90 \\
\hline Proposed_Tomato leaf & 2021 & 96.59 \\
\hline
\end{tabular}
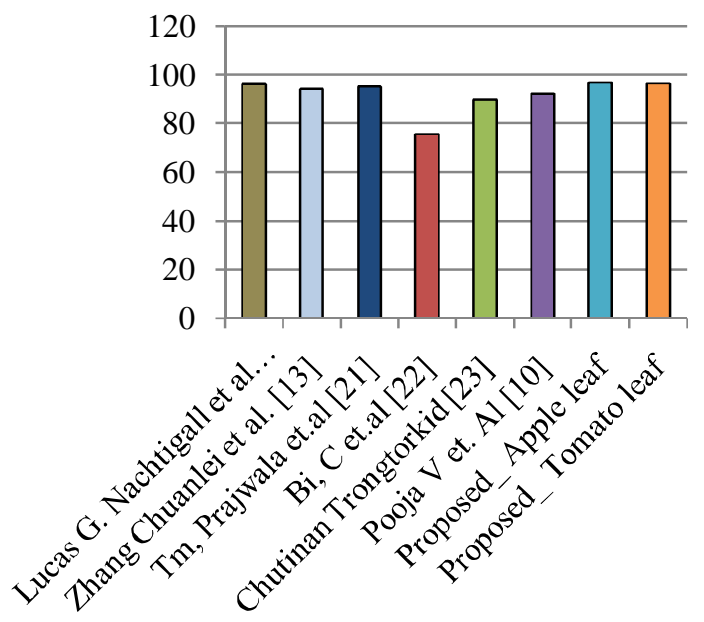

Figure 7: Comparative Analysis of Proposed Technique with Existing Techniques

The existing techniques achieved nearly $85 \%$ to $95 \%$ of accuracy for plant leaf disease detection, here we have taken a common plant leaf disease detection dataset. The reason is that no existing papers have the combination of apple and tomato disease detection, therefore the comparison is carried out for common datasets. The proposed CSSA with BiLSTM achieved $96.90 \%$ of accuracy for apple leaves disease detection, where the same method achieved $96.59 \%$ of accuracy for tomato leaves disease detection. Hence, the experimental analysis proves that the proposed process achieved better performance than existing practices.

\section{CONCLUSION}

The agricultural industry is still one of the most important sectors on which the majority of Indians rely. Detection of illnesses in these crops is thus crucial to economic progress. Tomatoes and apples are two major crops that are grown in enormous quantities. As a result, the resolution of this research is to detect and identify various illnesses in tomato and apple crops. To categorise tomato and apple leaf diseases from the Plant Village dataset, the suggested methodology employs a CSSA with Bi-LSTM network model. As part of the future work, other learning rates and optimizers could be employed to experiment with the suggested model. It could also contain experimenting with newer architectures to improve the model's performance on the train set. As a result, the aforementioned model can be used as a decision tool to assist and provision farmers in recognizing diseases that can be discovered in tomato plants. With an accuracy of 96 percent, the suggested technology can detect leaf diseases with low computational work.

\section{REFERENCES}

1. R. Kaundal, A. S. Kapoor, and G. P. S. Raghava, "Machine learning techniques in disease forecasting: a case study on rice blast prediction.," BMC Bioinformatics, vol. 7, p. 485, 2006.

2. C. Deisy and M. Francis, "Image Segmentation for Feature Extraction," no. June 2017, pp. 232257.

3. PiyushChaudhary, ShardaGodara, A. N. Cheeran, Anand K. Chaudhari, "Fast and Accurate Method for Leaf Area Measurement", International Journal of Computer Applications (0975 - 8887) Volume 49- No.9, July 2012.

4. MadhuJadon, RashiAgarwal, Raghuraj Singh, "An Easy Method for Leaf area Estimation Based on Digital Images", 2016 International Conference on Computational Techniques in Information and Communication Technologies (ICCTICT) IEEE 2016.

5. H. Sabrol, K. Satish, "Tomato Plant Disease Classification in Digital Images Using Classification Tree", International Conference on Communication and Signal Processing, April 6-8, IEEE 2016 India

6. Wan MohdFadzil W.M.N, Shah Rizam M.S.B, R. Jailani, Nooritawati M.T, "Orchid Leaf Disease Detection using Border Segmentation Techniques", 2014 IEEE Conference on Systems, Process and Control (ICSPC 2014), 12 - 14 December 2014, Kuala Lumpur, Malaysia.

7. P.R. Rothe, Dr. R. V. Kshirsagar, "Automated Extraction of Digital Images Features of three kinds of Cotton Leaf Diseases" , 2014 International Conference on Electronics, Communication and Computational Engineering (ICECCE) IEEE 2014.

8. M V Latte, SushilaShidnal, "Multiple Nutrient Deficiency Detection in Paddy Leaf Images using Color and Pattern Analysis", International Conference on Communication and Signal Processing, April 6-8, IEEE 2016 India.

9. Arya M S, Anjali K, and Mrs.DivyaUnni, "Detection of unhealthy plant leaves using Image 
Processing and Genetic Algorithm with Arduino", IEEE 2018.

10. Pooja V, Rahul Das, and Kanchan V, "Identification of Plant Leaf Diseases using Image Processing Techniques", IEEE 2017.

11. R.MeenaPrakash, G.P.Saraswathy, G.Ramalakshmi, K.H.Mangaleswari, T.Kaviya, "Detection of Leaf Diseases and Classification using Digital Image Processing", 2017 International Conference on Innovations in Information, Embedded and Communication Systems (ICIIECS) IEEE 2017.

12. L.G. Nachtigall, R.M. Araujo, G.R. Nachtigall, Classification of apple tree disorders using convolutional neural networks, in IEEE 28th International Conference on Tools with Artificial Intelligence (ICTAI), San Jose, CA, USA, 2016, pp. 472-476.

13. Z. Chuanlei, Z. Shanwen, Y. Jucheng, S. Yancui, C. Jia, Apple leaf disease identification using the genetic algorithm and correlationbased feature selection method, Int. J. Agric. Biol. Eng. 10 (2017), 74-83.

14. J.G.A. Barbedo, A new automatic method for disease symptom segmentation in digital photographs of plant leaves, Eur. J. Plant Pathol. 147 (2017), 349-364.

15. S. Kalaivani, S.P. Shantharajah, T. Padma, Agricultural leaf blight disease segmentation using indices based histogram intensity segmentation approach, Multimed. Tools Appl. (2019), $1-15$. https://link.springer.com/journal/11042/onlineFirst/ page/33

16. K. Golhani, S.K. Balasundram, G. Vadamalai, B. Pradhan, A review of neural networks in plant disease detection using hyperspectral data, Inf. Process. Agric. 5 (2018), 354-371.

17. I.F. Salazar-Reque, S.G. Huamán, G. Kemper, J. Telles, D. Diaz, An algorithm for plant disease visual symptom detection in digital images based on superpixels, Int. J. Adv. Sci. Eng. Inf. Technol. 9 (2019), 194-203.

18. S. Zhang, Z. You, X. Wu, Plant disease leaf image segmentation based on superpixel clustering and EM algorithm, Neural Comput. Appl. 31 (2019), 1225-1232.

19. YannLeCun et al. "Backpropagation applied to handwritten zip code recognition". In: Neural computation 1.4 (1989), pp. 541-551.

20. Mirjalili, S., Gandomi, A.H., Mirjalili, S.Z., Saremi, S., Faris, H. and Mirjalili, S.M., 2017. Salp Swarm Algorithm: A bio-inspired optimizer for engineering design problems. Advances in Engineering Software, 114, pp.163-191.

21. Tm, Prajwala, AllaPranathi, KandirajuSaiAshritha, Nagaratna B. Chittaragi, and Shashidhar G.
Koolagudi. "Tomato leaf disease detection using convolutional neural networks." In 2018 eleventh international conference on contemporary computing (IC3), pp. 1-5. IEEE, 2018.

22. Bi, C., Wang, J., Duan, Y., Fu, B., Kang, J.R. and Shi, Y., 2020. MobileNet based apple leaf diseases identification. Mobile Networks and Applications, pp.1-9.

23. ChutinanTrongtorkid, Part Pramokchon, "Expert System for Diagnosis Mango Diseases Using Leaf Symptoms Analysis", The 3rd International Conference on Digital Arts, Media and Technology (ICDAMT2018).

24. Dr. Paul P. Mathai, Aiswarya S , Aiswarya J.G. , Athira Saiju , Anagha Clare Antony, "SasyaSneha - An Approach for Plant Leaf Disease Detection", International Journal of Advanced Trends in Computer Science and Engineering, vol.8, No. 5, 2019.https://doi.org/10.30534/ijatcse/ 2019/38852019 